\title{
A Pilot Feasibility Study on a Single-Session Stabilization Group Psychotherapy for Adults with Acute Stress Symptoms
}

\author{
Dabin Kim, MD, $M S^{1}$, Daeho Kim, MD, PhD ${ }^{1}$, Hyunji Lee, $M A^{2}$, JiYoung Min, $M A^{3}$, Sungwon Roh, \\ $\mathrm{MD}, \mathrm{PhD}^{1}$
}

${ }^{1}$ Department of Psychiatry, Hanyang University College of Medicine, Seoul, ${ }^{2}$ Smile Center for Victims of Crime, Incheon, ${ }^{3}$ Center for Victims of Crime, Ministry of Justice, Seoul, Korea

Background: Although the field of psychology currently recommends trauma-focused cognitive behavioral therapy for early psychological intervention for acute traumatic stress, additional research is required for safe and efficient psychotherapy that can delivered to a broader population and within a brief period of time.

Methods: This pilot study examined the safety and feasibility of a single-session group stabilization intervention for individuals conducted at an average of two weeks after various types of traumatic events. Further development of DSM-5 mental disorders, such as post-traumatic stress disorder (PTSD) and others, was assessed at the six-month follow-up. A total of 38 participants with acute stress symptoms participated in a single-session 90-minute group psychotherapy, which consists of psychoeducation; identification of and coping with triggers; somatosensory grounding; and containment exercise.

Results: After six months, follow-up was conducted on 34 (89.5\%) patients, who completed the Structured Clinical Interview for DSM-5 Mental Disorders and the PTSD Checklist-5. One (2.9\%) participant met the current diagnosis of PTSD, whereas none met any other psychiatric diagnoses. A significant decrease was noted in PTSD scores between baseline and follow-up ( $t=7.4, d f=33, p<0.001$, Cohen's $d=1.27$ ) measured using the PTSD Checklist -5 .

Conclusion: The finding suggests that a single stabilization session can be used in a safe and efficient manner at of the acute stage of trauma.

Keywords Acute stress disorder; Group therapy; Posttraumatic stress disorder; Psychological trauma; Psychotherapy

\section{INTRODUCTION}

Early psychological interventions for trauma have received growing attention during the last two decades $[1,2]$. The aim of such interventions at acute stage of trauma is to decrease acute distress and further to prevent posttraumatic psychopathology such as post-traumatic stress disorder (PTSD) [3,4]. Acute stress response fosters lingering physical health problems such as pain, poor quality of well-being, and health care utilization and increased psychological outcomes such as PTSD, depression, and anxiety [5] Besides, subsequent PTSD may persist for years or decades and that those affected by chronic PTSD had to endure poor physical and psychological health and low quality of life [6,7].

Traditionally, psychological debriefing (also known as critical incident stress debriefing) was an intervention of popularity widely delivered to emergency service personnel and later to primary victims within 2 to 10 days after trauma; however, subsequent research showed that psychological debriefing not only failed to reduce of further development of PTSD but in some recipients it

Received October 13, 2021, Revised October 25, 2021, Accepted October 26, 2021

Correspondence: Daeho Kim, MD, PhD

Department of Psychiatry, Hanyang University College of Medicine, 222 Wangsimni-ro, Seongdong-gu, Seoul 04763, Korea

TEL +82-2-2290-8430 FAX +82-2-2298-2055 E-mail dkim9289@hanyang.ac.kr ORCID https://orcid.org/0000-0002-6834-6775

Copyright $\odot$ by Korean Society for Affective Disorders.

This is an Open Access article distributed under the terms of the Creative Commons Attribution Non-Commercial License (http://creativecommons.org/licenses/ by-nc/4.0/) which permits unrestricted non-commercial use, distribution, and reproduction in any medium, provided the original work is properly cited. 
interfered with natural recovery of acute distress [8-10].

This failure to prove the benefits of debriefing brought forth the basic questions such as whether early psychological intervention is indeed needed and if that is the case, who will be the good candidates for such interventions. According to UK NICE guideline, within one month after trauma, for those formally diagnosed with acute stress disorder (ASD) or those showing abovethreshold scores on self-report measures of PTSD, individual trauma-focused cognitive behavioral therapy (TFCBT) interventions were effective and recommended [11]. Equivalently, clinical guidelines of leading expert organizations such as VA/DoD [12], the World Health Organization [13], and Centre for Posttraumatic Mental Health in Australia [14] have recommended TF-CBT for those suffering from ASD. Meanwhile, only active monitoring was recommended for those exhibiting subthreshold PTSD symptoms [11] implying that target population should those with significant acute stress.

Despite current evidence-based recommendation in favor of TF-CBT, further development in the field of early interventions is needed for following reasons. First, it is about treatment dose, that is length of the intervention. Most TF-CBT studies had treatment sessions in the range of 5 to 12 sessions ( 7.5 to 18 hours), either delivered weekly or biweekly [2]. The treatment extends to the period of one month to three months after trauma which is in the time frame of acute PTSD. In addition, many with ASD symptoms will recover rapidly within one to three months after the traumatic events [15]. Thus, it is of question that the treatment should be as intensive in length as standard PTSD therapy and we need to look at brief version of therapy.

Second, some still do not respond to established treatment such as CBT; one study noted that CBT accelerated recovery of slow-remitting group (27\%) of ASD trajectories, but did not impact on the other two groups of rapid improving or non-remitting trajectories $(56 \%$ and $17 \%$, respectively) [15]. Vigorous research is needed to fill this gap and to develop effective psychological interventions as early intervention studies on other than CBT are very scarce [16].

Third, along with effectiveness safety requirements should be met, that is the therapy should be guided through 'first do no harm' principle. It is often suggested that recounting of trauma such as sharing one's own traumatic experience with others or exposure to traumatic materials from group members too early after the trauma may interfere with natural recovery as seen in psychological debriefing [8]. In similar vein, extensive exposure work at early phase after trauma may not be appropriate for some individuals with severe symptoms, unresolved past trauma, or marked ongoing stressors [17]. One emerging area in trauma treatment is emphasis of stabilization. It originally stems from phased approaches for complex PTSD, where initial period of intervention for establishing safety through psychoeducation, emotional regulation skills, stress management, social skill building, and cognitive restructuring before proceeding onto exposure to traumatic memory [18]. This principal also applies to interventions at immediate or mid-term post disaster or mass trauma, where the therapy should aim at promoting safety, calming, sense of efficacy, connectedness, and hope [19]. Likewise, psychological first aid, internationally endorsed approach in acute trauma aims at safety, calming, efficacy, connectedness, and hope after trauma exposure [20].

Recently, single or double-session brief interventions have emerged as promising early intervention strategies for preventing PTSD [2]. These include Group 512 psychological intervention model from critical incident stress debriefing [21], eye movement desensitization and reprocessing protocol for recent critical incidents [22] and brief dyadic therapy from CBT [23]. Although these interventions with some other single-session therapies $[24,25]$ stem from different treatment orientations, the therapy, as a rule, is organized with psychoeducation, coping or relaxation skills, and resource or motivational enhancement. In a previous study, Chung et al. [24] examined a single-session stabilizing group intervention of psychoeducation and affect regulation skills for 464 school children aged between 10 to 12 years after four days after trauma. The subjective distress was significantly decreased after treatment and $48 \%$ had a decrease of more than $50 \%$ of distress score from the traumatic experience. We used a further developed a single-session manualized group protocol for adults at acute stage of trauma [26] and the current study investigated the feasibility including safety and tolerability of such stabilization intervention for adults with acute stress symptoms. Our research question was whether this intervention could be delivered safely without distress, and development of post-traumatic psychopathology followed up after six months. 


\section{MATERIALS AND METHODS}

\section{Participants and procedure}

Recruitment was conducted through advertisement and referral at Handong University in Pohang, Seongdong Mental Health Center in Seoul, and Hanyang Universtiy Guri Hospital in Guri, Korea from March 2017 to August 2018. Inclusion criteria were 1) age between 18 to 70,2$)$ presentation within one month of trauma, 3) score of 3 or more the Primary Care PTSD Screen for DSM-5 (PC-PTSD-5) [27], and 4) ability to use the Korean language. Exclusion criteria were 1) intellectual disability and neurocognitive disorders, 2) severe medical condition, 3) psychotic illness, and 4) current psychiatric treatment.

This study was approved by the Research Ethics Board of Hanyang University Guri Hospital (IRB No. 2015-05-013), and informed consent was obtained from each participant. A final 38 survivors participated and completed the treatment, and $34(89.5 \%)$ were followedup after six months. Follow-up was performed by either telephone or face-to-face interviews that lasted approximately an hour; a self-report PTSD measure and structured diagnostic interview were administered. Participants received imbursement corresponding to 80 US dollars for the follow-up interview.

\section{Measures}

\section{1) Primary Care PTSD Screen for DSM-5}

The PC-PTSD-5 is a brief screening measure for DMS-5 PTSD and consists of one item for traumatic events and other five for clinical symptoms (nightmare, avoidance, hyperarousal, detachment, and guilt or blaming others). It has received psychometric investigations for the original English and the Korean translated versions $[27,28]$. One study with US veterans has recommended a score of three as optimally sensitive value (highest kappa score and acceptable specificity); Korean version also displayed the score of three as optimal cutoff point for detecting PTSD among civilians. Thus, eligibility to enter this study was the score three or more of the Korean version of the PC-PTSD-5.

\section{2) PTSD checklist-5}

The PTSD checklist (PCL)-5 is a 20 -item self-report measurement tool assessing the DSM- 5 version of PTSD symptoms. The original version has undergone psychometric evaluation and provided the excellent reliability and validity, particularly, when considering factorial validity fit with DSM-5 criteria [29]. The Korean version used in this study showed high internal consistency and good concurrent validity with other established PTSD measures [30]. We administered the Korean version of PCL-5 before the treatment and at follow-up after 6 months.

\section{3) Structured Clinical Interview for DSM-5 Mental Disorders}

The Structured Clinical Interview for DSM-5 Mental Disorders (SCID-5), Clinical version is a semi-structured interview that assesses DSM-5 mental disorders including mood disorders, psychotic disorders, substance use disorders, anxiety disorders, obsessive compulsive disorder, PTSD, attention deficit/hyperactive disorder,

Table 1. The contents of a single-session stabilization group therapy

\begin{tabular}{|c|c|c|}
\hline Protocol component & Minute & Description \\
\hline Introduction & 5 & $\begin{array}{l}\text { Introducing therapists and participants } \\
\text { Brief explanation of agenda and the session }\end{array}$ \\
\hline Psychoeducation & 20 & $\begin{array}{l}\text { Providing information on definition of trauma, common posttraumatic reactions and } \\
\text { their meanings, course, and treatment }\end{array}$ \\
\hline Identification of triggers & 10 & $\begin{array}{l}\text { Identification of external and internal triggers for intrusion or distress } \\
\text { Discussion of coping strategies }\end{array}$ \\
\hline Somatosensory grounding & 30 & $\begin{array}{l}\text { Teaching and exercising grounding skills to stay at present time and space by } \\
\text { sensory or motor modalities including visual, auditory, tactile sensation, or motor } \\
\text { movement }\end{array}$ \\
\hline Containment exercise & 20 & Development and exercising of visual imagery for containing posttraumatic distress \\
\hline Closure & 5 & Gaining feedback and explaining future plan \\
\hline
\end{tabular}


and adjustment disorder [31]. The Korean version of the SCID-5 was administered to 34 participants who were followed up after six months.

\section{Intervention}

Participants received a single 90-minute intervention in small group (2 to 6 members) presided over by one of three therapists: two clinical psychologists and a psychiatrist, all board specialized with at least 3 yearexperiences in trauma treatment practice. After the treatment, participants were asked to report any distress during after the session and overall experience with the therapy and none have reported the distress. The session was comprised of 1) introduction (5 min), 2) psychoeducation about trauma and reaction (20 min), 3) identifying triggers (10 min), 4) somatosensory grounding (30 min), 5) containment exercise (20 $\mathrm{min})$, and 6) closure (5 $\mathrm{min})$. The detailed description of the session including therapist' verbatim ac literatim can be found in the form of therapist protocol [26].

The intervention begins with greeting and introduction among therapists and participants followed by therapist's explanation of agenda and brief summary of the session. Next, therapist gives psychoeducation in the form of interactive lecture: definition of trauma; normalization and validation; common psychological and somatic reactions to trauma; meaning of such reaction; course of distress over the time; further help and resources. After questions from the participants if any, therapy moves onto identifying triggers. This phase begins with providing rationale, definition of triggers, identification of external and internal triggers, and discussing prior and future coping. Following somatosensory grounding involves teaching and exercising multisensory and motor grounding exercises: visual, auditory, tactile, and motor grounding. The next step is to create a visual imagery for containment of any posttraumatic distress and to rehearse it in the session. If any, positive emotion or sensation is associated, they will be enhanced through imagination. Final stage is closure where participants can share feedback and therapists provide future plan after the therapy (Table 1).

\section{Data analysis}

For comparison of PCL-5 scores between baseline and at follow-up, we conducted paired t-test and Cohen's d calculation for its effect size. Statistical significance was set at $\alpha=0.05$ (two-tailed).

\section{RESULTS}

\section{Sample characteristics}

Sociodemographic and clinical characteristics are shown in Table 2. Participants $(\mathrm{n}=38)$ had a mean 36.9 years of age (95\% confidence interval [CI], 31.0-42.8) and received treatment at a mean 14.6 days after trauma (95\% CI, 12.9-16.2). Eight (21.1\%) individuals had previous history of psychiatric treatment. The most common traumatic event was earthquake $(\mathrm{n}=18)$, followed by accidents $(\mathrm{n}=8)$, physical assaults $(\mathrm{n}=6)$, witnessed death

Table 2. Baseline characteristics of participants $(n=38)$

\begin{tabular}{|c|c|}
\hline Variable & Value \\
\hline \multicolumn{2}{|l|}{ Sex } \\
\hline Male & $10(26.3)$ \\
\hline Female & $28(73.7)$ \\
\hline \multicolumn{2}{|l|}{ Marital status } \\
\hline Married & $13(34.2)$ \\
\hline Not married & $25(65.8)$ \\
\hline \multicolumn{2}{|l|}{ Employment } \\
\hline Employed/students & $29(76.3)$ \\
\hline Unemployed/not working & $9(23.7)$ \\
\hline \multicolumn{2}{|l|}{ Education } \\
\hline Below high school & $6(15.8)$ \\
\hline High school graduate & $24(63.2)$ \\
\hline University or more & $8(21.1)$ \\
\hline \multicolumn{2}{|l|}{ Annual income (US dollars) } \\
\hline$<20,000$ & $4(10.5)$ \\
\hline $20,000-39,999$ & $21(55.3)$ \\
\hline$>40,000$ & $13(34.2)$ \\
\hline \multicolumn{2}{|l|}{ Index trauma } \\
\hline Earthquakes & $18(47.4)$ \\
\hline Accidents & $8(21.1)$ \\
\hline Physical assaults & $6(15.8)$ \\
\hline Witnessed death (loss) & $4(10.5)$ \\
\hline Traffic accidents & $2(5.3)$ \\
\hline \multicolumn{2}{|l|}{ Psychiatric history } \\
\hline Yes & $8(21.1)$ \\
\hline No & $30(78.9)$ \\
\hline Age $(y)$ & $36.9 \pm 17.9$ \\
\hline Days after trauma & $14.6 \pm 4.9$ \\
\hline
\end{tabular}

Values are presented as number (\%) or mean \pm standard deviation. 
$(n=4)$, and traffic accidents $(n=2)$.

\section{Assessment after six months}

Among 34 participants who were followed-up at sixmonth, one $(2.9 \%)$ was diagnosed as full PTSD. Three participants met the criteria for current major depressive disorder and one for current PTSD, that preceded the onset of index trauma. Current adjustment disorder was diagnosed in one participant due to unrelated stressor after the index trauma. The PCL-5 scores significantly decreased after six months: initial value was mean score of 31.5 (standard deviation $[\mathrm{SD}]=14.3$ ) and follow-up value at six months was mean score of $12.5(\mathrm{SD}=11.6)$ $(\mathrm{t}=7.4, \mathrm{df}=33, \mathrm{p}<0.001)$. Effect size was Cohen's $\mathrm{d}=1.27$ (95\% CI, 0.81-1.72).

\section{DISCUSSION}

In this study, none of those who received a single-session stabilization group stabilization have reported distress during or after treatment. The treatment was safely delivered to those with acute stress symptoms. This is consistent with 'do no harm' principle of acute trauma and the current consensus for early trauma intervention that psychological intervention at this stage should not focus on the catharsis and exploration of trauma, that but stabilization of affect and coping skill building should be provided [19,32]. It is not known how common exacerbation of symptoms during psychotherapy with ASD; one study noted that $14.7 \%-28.6 \%$ of participants in a TF-CBT trial have reported that they had such experience during sessions, although exacerbation during PTSD treatment did not last to the end of treatment [33].

Six months after that therapy, PTSD symptoms significantly decreased after six months and one participant (2.9\%) had developed PTSD after therapy. No other psychopathological conditions were found related to the index trauma including adjustment disorder, depressive disorder, anxiety disorder, substance use disorder and etc. Our findings do confirm the effectiveness of single session therapy to decrease ASD symptoms and to prevent further development of PTSD, because one cannot rule out the effect of natural recovery for ASD symptoms. Nonetheless, our rate $(2.6 \%)$ is much lower compared to the $31 \%$ PTSD rate at seven months after trauma among emergency visitors with ASD [15].
Although the baseline and follow-up prevalence of PTSD varies widely across studies [34], the improvement from acute stress over time is clear as seen in a systemic review; median prevalence of PTSD was $28.9 \%$ at one month but at six months was $14.9 \%$ among PTSD trajectories from 35 populations [35]. However, longitudinal change that is decrease in PTSD symptoms and prevalence does not seem to be robust; a multi-site study of traumatically injured patients have found that the rate of PTSD at 3, 12, and 24 months later remained constantly over time [36].

Our study investigated any development of other posttraumatic psychiatric disorders in addition to PTSD at follow-up. These comorbid or independent conditions are often neglected in the field of trauma research. Approximately $80 \%$ of individuals with PTSD have at least one concurrent psychiatric disorder including affective disorders, anxiety disorders, substance abuse, and dissociative disorders [37]. One longitudinal study found that adjustment disorder was the most common mental illness arisen after trauma exposure [38]. More complete coverage of posttraumatic psychopathologies and no development of such conditions at six-month assessment further add to the strength of our intervention.

Moreover, compared to multiple-session psychotherapy, current intervention is more efficient; the brief duration and group format can allow treatment to be provided to a larger population and in situations with limited treatment resources often is the case at aftermath of trauma. Current status of TF-CBT as early intervention is delivered with a range of 4.5 to 18 hours, often extending to one to three months after trauma [2].

Overall, our fining supports use of a single-session intervention for acute phase of trauma. Single-session early interventions that emerged in recent literature targets different populations including military rescue workers [21], technical disaster survivors [22], and disaster volunteers [39], school children witnessing deaths [24], and children inpatients [25]. However, they have very similar composition of the session involving psychoeducation (giving information on trauma reactions and normalization of experience), teaching coping skills to distress (affect regulation or relaxation exercises). Some interventions add resource building or positive psychology.

Several limitations of this study should be noted. First, the uncontrolled nature of this study and small sample size require a caution for interpretation and generalization of results. By design, one cannot rule out that im- 
provement by natural course of time. In fact, in a waitlist controlled study, trauma-exposed adults who received a single-session psychoeducational intervention showed higher symptoms than control group of no treatment [40]. Thus, a controlled clinical trial is essential to prove effectiveness of current intervention. Second, participants in this study had heterogenous trauma types and diverse demographic characteristics that may influence the response and acceptability of therapy. Further, subgroup analyses or study on mediating factors are needed with larger sample. Third, a longer follow-up period, such 12 months, may provide better understating of the clinical course of participants. Fourth, this study lacks an immediate post-treatment evaluation and use of a scale specific for ASD. Nonetheless, this is a pilot study that examined a single intervention for acute trauma survivors and confirmed safe delivery to those suffering from acute stress symptoms. Future study should investigate effectiveness and tolerability with a larger sample size at various stages of time after trauma.

\section{CONFLICTS OF INTEREST}

The authors have nothing to disclose.

\section{ACKNOWLEDGEMENTS}

This study was supported by the Korean Mental Health Technology R\&D Project, Ministry of Health \& Welfare, Republic of Korea (HM15C1058).

\section{ORCID}

Dabin Kim
https://orcid.org/0000-0002-9714-9041
Daeho Kim
https://orcid.org/0000-0002-6834-6775
Hyunji Lee
https://orcid.org/0000-0001-7470-9416
Ji Young Min
https://orcid.org/0000-0002-7912-6153
Sungwon Roh
https://orcid.org/0000-0003-4557-3542

\section{REFERENCES}

1. Kearns MC, Ressler KJ, Zatzick D, Rothbaum BO. Early interventions for PTSD: a review. Depress Anxiety 2012; 29:833-42

2. O’Donnell ML, Pacella BJ, Bryant RA, Olff M, Forbes D. Early intervention for trauma-related psychopathology. In: Forbes D, Bisson JI, Monson CM, Berliner L, eds. Effective treatments for PTSD. NewYork: Guilford Press; 2020. p. 117-36.

3. Howlett JR, Stein MB. Prevention of trauma and stressorrelated disorders: a review. Neuropsychopharmacology 2016;41:357-69.

4. Roberts NP, Kitchiner NJ, Kenardy J, Lewis CE, Bisson JI. Early psychological intervention following recent trauma: a systematic review and meta-analysis. Eur J Psychotraumatol 2019; 10:1695486.

5. Garfin DR, Thompson RR, Holman EA. Acute stress and subsequent health outcomes: a systematic review. J Psychosom Res 2018;112:107-13.

6. Hidalgo RB, Davidson JR. Posttraumatic stress disorder: epidemiology and health-related considerations. J Clin Psychiatry 2000;61 Suppl 7:5-13.

7. Steinert C, Hofmann M, Leichsenring F, Kruse J. The course of PTSD in naturalistic long-term studies: high variability of outcomes. A systematic review. Nord J Psychiatry 2015;69:483-96.

8. van Emmerik AA, Kamphuis JH, Hulsbosch AM, Emmelkamp PM. Single session debriefing after psychological trauma: a meta-analysis. Lancet 2002;360:766-71.

9. Rose S, Bisson J, Wessely S. A systematic review of singlesession psychological interventions ('debriefing') following trauma. Psychother Psychosom 2003;72:176-84.

10. McNally RJ, Bryant RA, Ehlers A. Does early psychological intervention promote recovery from posttraumatic stress? Psychol Sci Public Interest 2003;4:45-79.

11. National Institute for Health and Care Excellence. NICE guideline. Post-traumatic stress disorder. National Institute for Health and Care Excellence; 2018.

12. Bernardy NC, Hoge CW, Friedman MJ, Riggs DS, Schnurr PP. VA/DoD clinical practice guidelines. Management of posttraumatic stress disorder and acute stress reaction 2017. Washington, D.C.: U.S. Department of Veterans Affairs; 2017.

13. World Health Organization. WHO guidelines on conditions specifically related to stress. Geneva: World Health Organization; 2013.

14. Australian Government National Health and Medical Research Council. Australian guidelines for the prevention and treatment of acute stress disorder, posttraumatic stress disorder and complex PTSD. Australian Government National Health and Medical Research Council; 2020.

15. Galatzer-Levy IR, Ankri Y, Freedman S, Israeli-Shalev Y, Roitman P, Gilad M, et al. Early PTSD symptom trajecto- 
ries: persistence, recovery, and response to treatment: results from the Jerusalem Trauma Outreach and Prevention Study (J-TOPS). PLoS One 2013;8:e70084.

16. Birur B, Moore NC, Davis LL. An evidence-based review of early intervention and prevention of posttraumatic stress disorder. Community Ment Health J 2017;53:183-201.

17. Bryant RA, Harvey AG. Acute stress disorder: a handbook of theory, assessment, and treatment. Washington, D.C.: American Psychological Association; 2000.

18. Cloitre M, Courtois CA, Ford JD, Green BL, Alexander $\mathrm{P}$, Briere J, et al. The ISTSS expert consensus treatment guidelines for complex PTSD in adults. Chicago: International Society for Traumatic Stress Studies; 2012.

19. Hobfoll SE, Watson P, Bell CC, Bryant RA, Brymer MJ, Friedman MJ, et al. Five essential elements of immediate and mid-term mass trauma intervention: empirical evidence. Psychiatry 2007;70:283-315; discussion 316-69.

20. World Health Organization. Psychological first aid: guide for field workers. Geneva: World Health Organization; 2011.

21. Wu S, Zhu X, Zhang Y, Liang J, Liu X, Yang Y, et al. A new psychological intervention: "512 Psychological Intervention Model" used for military rescuers in Wenchuan Earthquake in China. Soc Psychiatry Psychiatr Epidemiol 2012;47:1111-9.

22. Jarero I, Uribe S, Artigas L, Givaudan M. EMDR protocol for recent critical incidents: a randomized controlled trial in a technological disaster context. J EMDR Pract Res 2015;9:166-73.

23. Brunet A, Des Groseilliers IB, Cordova MJ, Ruzek JI. Randomized controlled trial of a brief dyadic cognitivebehavioral intervention designed to prevent PTSD. Eur J Psychotraumatol 2013;4:21572.

24. Chung CY, Kim D, Kim SH, Bae H, Lee K, Go B. Treatment response of affect regulation group therapy for recently traumatized school children. J Korean Neuropsychiatr Assoc 2014;53:171-7.

25. Fukuchi N. Psychoeducation for children in a psychiatric ward in the immediate aftermath of the 2011 earthquake and tsunami in Japan. Intervention 2020;18:85-91.

26. Kim D, Lee H, Min J. Basic treatment protocol manual for disaster surviors. Seoul: SIID communication; 2019.

27. Prins A, Bovin MJ, Smolenski DJ, Marx BP, Kimerling $\mathrm{R}$, Jenkins-Guarnieri MA, et al. The primary care PTSD screen for DSM-5 (PC-PTSD-5): development and evaluation within a veteran primary care sample. J Gen Intern Med 2016;31:1206-11.

28. Jung YE, Kim D, Kim WH, Roh D, Chae JH, Park JE. A brief screening tool for PTSD: validation of the Korean version of the primary care PTSD screen for DSM-5 (KPC-PTSD-5). J Korean Med Sci 2018;33:e338.
29. Blevins CA, Weathers FW, Davis MT, Witte TK, Domino JL. The posttraumatic stress disorder checklist for DSM-5 (PCL-5): development and initial psychometric evaluation. J Trauma Stress 2015;28:489-98.

30. Kim JW, Chung HG, Choi JH, So HS, Kang SH, Kim DS, et al. Psychometric properties of the Korean version of the PTSD checklist-5 in elderly Korean veterans of the Vietnam war. Anxiety Mood 2017;13:123-31.

31. First MB, Williams JB, Karg RS, Spitzer RL. User's guide for the SCID-5-CV structured clinical interview for DSM-5 disorders: clinician version. Arlington: American Psychiatric Association; 2016.

32. Berkowitz S, Bryant R, Brymer M, Hamblen J, Jacobs A, Layne C, et al. Skills for psychological recovery: field operations guide. Washington D.C.: U.S. Department of Veterans Affairs; 2010.

33. Larsen SE, Wiltsey Stirman S, Smith BN, Resick PA. Symptom exacerbations in trauma-focused treatments: associations with treatment outcome and non-completion. Behav Res Ther 2016;77:68-77.

34. Qi W, Ratanatharathorn A, Gevonden M, Bryant R, Delahanty D, Matsuoka Y, et al. Application of data pooling to longitudinal studies of early post-traumatic stress disorder (PTSD): the International Consortium to Predict PTSD (ICPP) project. Eur J Psychotraumatol 2018;9:1476442.

35. Santiago PN, Ursano RJ, Gray CL, Pynoos RS, Spiegel D, Lewis-Fernandez R, et al. A systematic review of PTSD prevalence and trajectories in DSM-5 defined trauma exposed populations: intentional and non-intentional traumatic events. PLoS One 2013;8:e59236.

36. Bryant RA, O’Donnell ML, Creamer M, McFarlane AC, Silove D. A multisite analysis of the fluctuating course of posttraumatic stress disorder. JAMA Psychiatry 2013;70: 839-46.

37. Brady KT. Posttraumatic stress disorder and comorbidity: recognizing the many faces of PTSD. J Clin Psychiatry 1997;58 Suppl 9:12-5.

38. O’Donnell ML, Alkemade N, Creamer M, McFarlane AC, Silove D, Bryant RA, et al. A longitudinal study of adjustment disorder after trauma exposure. Am J Psychiatry 2016;173:1231-8.

39. Okanoya J, Kimura R, Mori M, Nakamura S, Somemura H, Sasaki N, et al. Psychoeducational intervention to prevent critical incident stress among disaster volunteers. Kitasato Med J 2015;45:62-8.

40. Ghafoori B, Fisher D, Korosteleva O, Hong M. A randomized, controlled pilot study of a single-session psychoeducation treatment for urban, culturally diverse, traumaexposed adults. J Nerv Ment Dis 2016;204:421-30. 